\title{
Emission and Regulation of Volatile Chemicals from Globe Amaranth Flowers
}

\author{
Yifan Jiang \\ College of Horticulture, Northwest Agricultural and Forestry University, Yangling, Shaanxi 712100, \\ China; and the Department of Plant Sciences, University of Tennessee, Knoxville, TN 37996 \\ Nan Zhao \\ Department of Plant Sciences, University of Tennessee, Knoxville, TN 37996
}

Fei Wang

College of Horticulture, Northwest Agricultural and Forestry University, Yangling, Shaanxi 712100, China

\author{
Feng Chen ${ }^{1}$ \\ Department of Plant Sciences, University of Tennessee, 2431 Joe Johnson Drive, Knoxville, TN 37996
}

AdDitional INDEX words. Gomphrena globosa, floral scent, headspace, salicylic acid, jasmonic acid, gas chromatographymass spectrometry, GC-MS

\begin{abstract}
Aвstract. Volatile chemicals emitted from the flowers of globe amaranth (Gomphrena globosa) were collected using a dynamic headspace technique and analyzed using gas chromatography-mass spectrometry. Among the four globe amaranth cultivars analyzed, Fireworks was the highest producer of floral volatiles. The flowers of the other three cultivars, Las Vegas White, Las Vegas Pink, and Las Vegas Purple, emit less volatiles, both qualitatively and quantitatively, than 'Fireworks'. 'Fireworks' was chosen for detailed characterization of regulation of floral volatile emission. A diurnal pattern of emission of floral volatiles was observed from the flowers of 'Fireworks'. In addition, the emission pattern was not significantly affected by light, suggesting that the circadian clock plays a major role in the regulation of volatile emission. The emission of floral volatiles from 'Fireworks' flowers that were treated with several chemicals was also analyzed. The treatment with silver thiosulphate, an ethylene inhibitor, led to enhanced emission of total volatiles. In contrast, the treatments with salicylic acid and jasmonic acid led to enhanced emission of total floral volatiles at $4 \mathrm{~h}$ but reduced emission at $24 \mathrm{~h}$ after the treatment. Biochemical pathways leading to the production of the major floral volatiles of globe amaranth are proposed and partly validated by cluster analysis of floral volatiles emitted from 'Fireworks' flowers under various conditions. The implications of the results of this study to the understanding of the reproductive biology of globe amaranth and the breeding of novel globe amaranth cultivars are discussed.
\end{abstract}

Globe amaranth is an important horticultural species native to Central America. It features globe-shaped, clover-like flowerheads, $4 \mathrm{~cm}$ long at maturity. The true flowers within the flowerheads are tiny and inconspicuous. As a tropical annual plant, globe amaranth continually blooms throughout summer and early fall. Thus, it has been a favorite landscape plant in annual beds, rock gardens, and garden borders. After being removed from the plant, the papery-textured flowers of globe amaranth can retain their color and shape for a long time. Therefore, globe amaranth has also been popular as a cut flower and a dried flower (Cocozza Talia, 1993; Lourdusamy et al., 2002). Continuous germplasm selection and breeding have led to the production of many cultivars of globe amaranth such as those varying in flower colors that include purple, lavender, magenta, red, orange, and white.

Despite the fact that globe amaranth is an important annual flower plant and its flowers have been the subject of many biological studies, little, if anything, is known about its floral

Received for publication 13 Sept. 2010. Accepted for publication 22 Oct. 2010. We thank Mr. Doug Lemley of the Ball Horticulture Company for sharing information with us about the reproductive biology and breeding of globe amaranth. We also thank Ms. Lori Osburn in the Department of Plant Sciences, University of Tennessee, for her critical reading of the manuscript.

${ }^{1}$ Corresponding author. E-mail: fengc@utk.edu. volatile chemistry. Globe amaranth is an outcrossing species. Flowers of globe amaranth are visited by butterflies, bees, and other insects. For plants that are cross-pollinated, floral volatiles often play an important role in their reproductive success by functioning as a cue to attract pollinators (Effmert et al., 2005). In addition, the flowers of globe amaranth have been used as a type of herbal medicine (Niu et al., 2009), implying that they may contain essential oils. Based on the hypothesis that globe amaranth flowers produce and emit volatiles, the first objective of this study was to determine what volatile compounds are emitted from the flowers of globe amaranth.

Another aspect of our interest on floral volatile chemistry of globe amaranth lies in the regulation of floral volatile emission. Emission of floral volatiles of some plants has been shown to exhibit a diurnal pattern (Matile and Altenburger, 1988). For example, emission of methyl benzoate, one of the most abundant scent compounds of bee-pollinated snapdragon (Antirrhinum majus) flowers, occurs in a rhythmic manner with the maximum emission coinciding with the foraging activity of bumblebees (Bombus spp.) (Kolosova et al., 2001). Thus, the second objective of this study was to determine whether the emission of floral volatiles of globe amaranth display a diurnal pattern; and, if so, whether the diurnal pattern is regulated by light and/or the circadian clock. 
Ethylene is an important plant hormone involved in many aspects of plant biology, including flower development and senescence. It has been shown to be involved in the regulation of floral volatile emissions of certain plants (Underwood et al., 2005). Because the flowers of globe amaranth senesce at a very slow rate, we are interested in determining whether ethylene is involved in regulating floral volatile emission of this species. In addition to attracting pollinators, some floral volatiles have a defense role to deter herbivorous insects and other unwanted visitors (Raguso, 2004). For example, nicotin emitted from the flowers of tobacco (Nicotiana attenuate) has a defense role (Euler and Baldwin, 1996). The jasmonic acid (JA) pathway has been shown to play a critical role in production and emission of defense plant volatiles from vegetative tissues (Howe, 2004). In addition, salicylic acid (SA) is also involved in the production and release of some defense volatile compounds (Shulaev et al., 1997; Zhao et al., 2010). However, little is known on whether the JA and SA signaling pathways play a role in regulating floral volatiles. The third objective of this study was to determine whether silver thiosulphate (STS), an ethylene inhibitor, JA, and SA can affect the emission of volatiles from the flowers of globe amaranth.

In this study, a dynamic headspace technique was used to collect volatiles released from the flowers of globe amaranth and the volatiles were analyzed using gas chromatographymass spectrometry (GC-MS). First, four cultivars of globe amaranth, Las Vegas White, Las Vegas Pink, Las Vegas Purple, and Fireworks, that have flower colors of white, pink, purple, and pink, respectively, were analyzed and compared for their floral volatile profiles. Then 'Fireworks' was chosen for a detailed study of regulation of floral volatile emission, including the impact of light, STS, JA, and SA. Lastly, biochemical pathways leading to the production of predominant floral volatiles from globe amaranth are proposed and the implications to globe amaranth breeding are discussed.

\section{Materials and Methods}

Plant Materials. Four cultivars of globe amaranth, Fireworks, Las Vegas White, Las Vegas Pink, and Las Vegas Purple, were used in the study. Seeds were obtained from the Ball Horticulture Co. (Chicago, IL) and planted in the University of Tennessee Gardens, Knoxville, in Spring 2009. The plants started to flower in August and continually bloomed until November. Flower samples were collected at $0900 \mathrm{HR}$, transferred to the laboratory, and analyzed on the same day from late September to early Oct. 2009. Individual inflorescences with a pedicle $\approx 6 \mathrm{~cm}$ long were detached from the plant, placed in a flask filled with distilled water, and transported to the laboratory for volatile analysis.

Headspace volatile collection. Volatiles emitted from flowers were collected in an open headspace sampling system (Analytical Research Systems, Gainesville, FL) as previously reported (Chen et al., 2009a; Zhuang et al., 2008). Six detached inflorescences in a single flask with $\approx 150 \mathrm{~mL}$ of distilled water were placed in a glass chamber $(10 \mathrm{~cm}$ diameter, $30 \mathrm{~cm}$ tall $)$ covered with a removable o-ring snap lid. Charcoal-purified air entered the chamber at a flow rate of $0.8 \mathrm{~L} \cdot \mathrm{min}^{-1}$ from the top through a Teflon hose. Volatiles were collected for $4 \mathrm{~h}$ by pumping air from the chamber through a SuperQ volatile collection trap (Analytical Research Systems) and eluted using $100 \mu \mathrm{L}$ methylene chloride containing 1-octanal $(0.003 \% \mathrm{w} / \mathrm{v})$ as an internal standard.
DETERMINATION OF EMISSION DYNAMICS OF FLORAL VOLATILE OF 'FIREWORKs'. To determine the temporal emission dynamics of floral volatiles from 'Fireworks', detached inflorescences were subject to volatile collection under two light regimes: a normal light/dark (12/12 h) cycle and continuous darkness. Headspace collections were performed continuously with one elution every $4 \mathrm{~h}$ for $48 \mathrm{~h}$.

The EFFECT OF SILVER THIOSUlPHATE, JASMONIC ACID, AND SALICYLIC ACID ON THE EMISSION OF THE FLORAL VOLATILES IN 'Fireworks'. Detached inflorescences of 'Fireworks' were placed in flasks filled with either distilled water (control) or solutions containing STS, SA, or JA. The concentrations of STS, SA, and JA were $0.2 \mathrm{mM}, 5 \mathrm{mM}$, and $10 \mu \mathrm{M}$, respectively, which are physiologically relevant based on previous studies (Chen et al., 2003; Sexton et al., 1995; Yang et al., 2006; Zhao et al., 2009). Headspace collections were performed from 0 to $4 \mathrm{~h}$ and from 20 to $24 \mathrm{~h}$ after the initiation of each treatment. SA, JA, and STS were purchased from Sigma-Aldrich (St. Louis, MO).

GAS CHROMATOGRAPHY-MASS SPECTROMETRY ANALYSIS. Floral volatiles were analyzed by a gas chromatograph (17A; Shimadzu, Columbia, MD) coupled to a quadrupole mass selective detector (QP5050A; Shimadzu). Separation was performed on a column $30 \mathrm{~m} \times 0.25 \mathrm{~mm}$ i.d. $\times 0.25 \mu \mathrm{m}$ thick (Restek SHR5XLB; Shimadzu). Helium was the carrier gas (flow rate of $5 \mathrm{~mL} \cdot \mathrm{min}^{-1}$ ), a splitless injection (injection temperature $250{ }^{\circ} \mathrm{C}$ ) was used, and a temperature gradient of $6{ }^{\circ} \mathrm{C} \cdot \mathrm{min}^{-1}$ from $60{ }^{\circ} \mathrm{C}(3-\mathrm{min}$ hold $)$ to $300{ }^{\circ} \mathrm{C}$ was applied. Products were identified using the National Institute of Standards and Technology mass spectral database and by comparison of retention times and mass spectra with authentic reference compounds if available. Quantification was performed based on peak areas of mass chromatograms.

HiERARCHICAL CLUSTERING OF THE MAJOR COMPOUNDS. Emission rates of major volatile compounds from the flowers of 'Fireworks' were used in a hierarchical cluster analysis, which was conducted using average linkage analysis in Cluster 3.0 software (Version 1.39; Stanford University, Palo Alto, CA). Heat maps were created using Java TreeView 1.60 software (Stanford University, Stanford, CA).

Statistical Analysis. Analyses of volatile quantification with treatment of various solutions were conducted using SAS (Version 8.02; SAS Institute, Cary, NC) based on three biological replicates (three independent flower samples) and two technical replicates (two runs of the same volatile sample on GC-MS) and SEs were calculated for all mean values. Levels of significance were calculated using Student's $t$ test at $P \leq 0.05$.

\section{Results}

EMISSION OF FLORAL VOLATILE FROM FOUR CULTIVARS OF GLOBE AMARANTH. One major difference among cultivars of globe amaranth is the color of their flowers. In this study, we compared the floral volatile chemistry of four cultivars, Fireworks, Las Vegas White, Las Vegas Pink, and Las Vegas Purple, which have flower colors of pink, white, pink and purple, respectively. 'Fireworks' showed the highest level of emission among the four cultivars with a total emission rate of $1605.5 \mathrm{ng} \cdot \mathrm{h}^{-1}$ per inflorescence. The total emission rates of the other three cultivars were similar compared with each other but much lower than that of 'Fireworks'. 'Las Vegas White', 'Las Vegas Pink', and 'Las Vegas Purple' emitted floral volatiles at the rate of 75.4, 71.5, and $67.7 \mathrm{ng} \cdot \mathrm{h}^{-1}$ per inflorescence, respectively (Fig. 1). 
Being the highest emitter of floral volatiles among the four cultivars, Fireworks also emitted most volatile compounds. A total of 13 volatiles was detected from 'Fireworks' flowers. In contrast, five, five, and six volatiles were detected from the flowers of 'Las Vegas White', 'Las Vegas Pink', and 'Las Vegas Purple', respectively (Table 1). Floral volatiles of 'Fireworks' were composed of terpenoids and terpenoid derivatives $[(\mathrm{E})-\beta$-ocimene, (E)- $\beta$-caryophyllene, geranyl acetone, geranyl propionate and geranyl isovalerate], aromatics (benzaldehyde, benzyl alcohol, benzyl isovalerate, and benzyl tiglate), and fatty acid derivatives (nonanal, decanal, 3-hexen1-ol acetate, and 4,8,12-tetradecatrienal, 5,9,13-trimethyl). (E)$\beta$-ocimene was the most abundant compound, accounting for approximately half of the total emission.

Four compounds were common among all four cultivars: nonanal, decanal, geranyl acetone, and 4,8,12-tetradecatrienal, 5,9,13-trimethyl. For 'Las Vegas White', 'Las Vegas Pink', and 'Las Vegas Purple', 4,8,12-tetradecatrienal, 5,9,13-trimethyl was the most abundant floral volatile. For terpenoids, (E)$\beta$-caryophyllene was detected from 'Las Vegas White' and (E)- $\beta$-ocimene was detected from 'Las Vegas Purple'. No terpenoid volatile was detected from 'Las Vegas Pink'. In con-

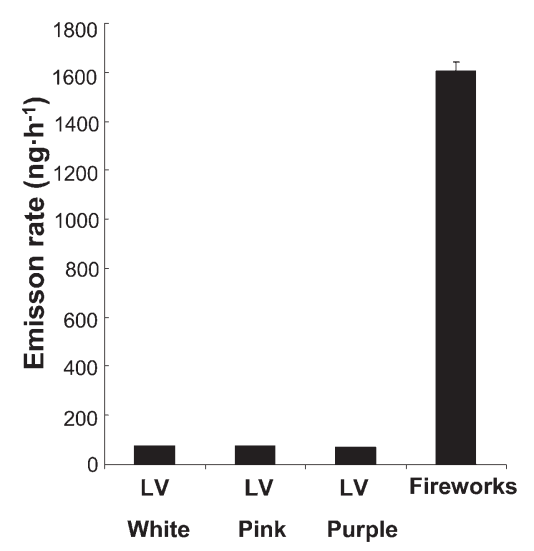

Fig. 1. Emission rates of total floral volatiles from four globe amaranth cultivars, Las Vegas White (LV White), Las Vegas Pink (LV Pink), Las Vegas Purple (LV Purple), and Fireworks. The values are the average of three independent measurements. The emission rates are presented as nanograms per hour per inflorescence. tract, benzaldehyde was detected from 'Las Vegas Pink' flowers but not from the other two cultivars (Table 1).

EMission dYNAMics OF FLORAL VOLATILE OF 'FirewORKS'. To examine the emission dynamics of floral volatiles, volatiles emitted from 'Fireworks' flowers were continuously collected every $4 \mathrm{~h}$ across two photoperiods (12/12 h of light/dark). The total emission rate of volatile compounds showed a diurnal pattern with the maximum emission occurring at late afternoon and the lowest level of emission occurring at the night (Fig. 2A). The emission dynamics of four major volatile compounds, including (E)- $\beta$-ocimene, benzaldehyde, geranyl isovalerate, and benzyl tiglate, also were analyzed. All four volatiles displayed an emission pattern similar to that of total emission (Fig. 2B-E).

To determine the mechanism that regulates the diurnal emission of floral volatiles, 'Fireworks' flowers were placed under continuous darkness. Volatiles emitted from flowers were continuously collected every $4 \mathrm{~h}$ for $2 \mathrm{~d}$. The emission rate of total floral volatiles and individual major compounds under a constant dark treatment showed similar patterns as those observed from flowers under normal light/dark cycles (Fig. 2A-E).

The EFFECTS OF SILVER THIOSUlPHATE, JASMONIC ACID, AND SALICYLIC ACID ON EMISSION OF FLORAL VOLATILE OF 'Fireworks'. The total emission rate of volatile compounds from the STS treatment was higher than control at both 4 and $24 \mathrm{~h}$ after the beginning of the treatment (Fig. 3A). STS showed different effects to the emission of individual compounds in 'Fireworks'. Among the six major compounds analyzed that include (E)- $\beta$-ocimene, benzyl isovalerate, geranyl propionate, geranyl isovalerate, and benzyl tiglate, three compounds [(E)$\beta$-ocimene, geranyl propionate and geranyl isovalerate] showed higher emission from STS-treated flowers than controls at both 4 and $24 \mathrm{~h}$ after the treatment. The emission of the other three compounds was not significantly affected by the STS treatment at both time points (Fig. 3B-C).

The total emission rates of volatile compounds from the SAand JA-treated flowers of 'Fireworks' were higher than the control at $4 \mathrm{~h}$ after the treatment. In contrast, the emission rates of treated flowers were lower that those of control at $24 \mathrm{~h}$ after the treatment (Fig. 3A). For individual compounds, the emission rates of benzyl tiglate, geranyl propionate, and geranyl

Table 1. Floral volatiles and their emission rates from four cultivars of globe amaranth.

\begin{tabular}{|c|c|c|c|c|}
\hline \multirow[b]{2}{*}{ Floral volatile } & Fireworks & Las Vegas White & Las Vegas Pink & Las Vegas Purple \\
\hline & \multicolumn{4}{|c|}{ Emission rate $\left[\right.$ mean $\pm \mathrm{sD}\left(\mathrm{ng} \cdot \mathrm{h}^{-1}\right.$ per inflorescence $\left.)\right]$} \\
\hline Benzaldehyde & $340.0 \pm 22.6^{z}$ & $\mathrm{ND}^{\mathrm{y}}$ & $20.4 \pm 1.7$ & ND \\
\hline 3-hexen-1-ol acetate & $10.4 \pm 0.9$ & ND & ND & $7.3 \pm 0.4$ \\
\hline Benzyl alcohol & $42.5 \pm 2.6$ & ND & ND & ND \\
\hline Nonanal & $12.2 \pm 2.3$ & $5.0 \pm 0.7$ & $5.7 \pm 0.5$ & $10.6 \pm 0.3$ \\
\hline Decanal & $6.8 \pm 0.6$ & $14.7 \pm 1.1$ & $5.0 \pm 0.7$ & $8.6 \pm 0.4$ \\
\hline (E)- $\beta$-caryophyllene & $7.8 \pm 1.1$ & $4.8 \pm 0.7$ & ND & ND \\
\hline Geranyl acetone & $13.7 \pm 1.8$ & $14.7 \pm 1.4$ & $13.8 \pm 1.2$ & $8.0 \pm 0.7$ \\
\hline Geranyl propionate & $65.1 \pm 1.9$ & ND & ND & ND \\
\hline Geranyl isovalerate & $91.8 \pm 3.2$ & ND & ND & ND \\
\hline Benzyl tiglate & $115.6 \pm 13.7$ & ND & ND & ND \\
\hline 4,8,12-tetradecatrienal, 5,9,13-trimethyl & $5.1 \pm 0.4$ & $36.3 \pm 2.8$ & $26.5 \pm 2.9$ & $26.1 \pm 1.6$ \\
\hline
\end{tabular}

${ }^{\mathrm{z}}$ Means of three biological replicates and two technical replicates were presented.

${ }^{\mathrm{y}}$ Not detected. 

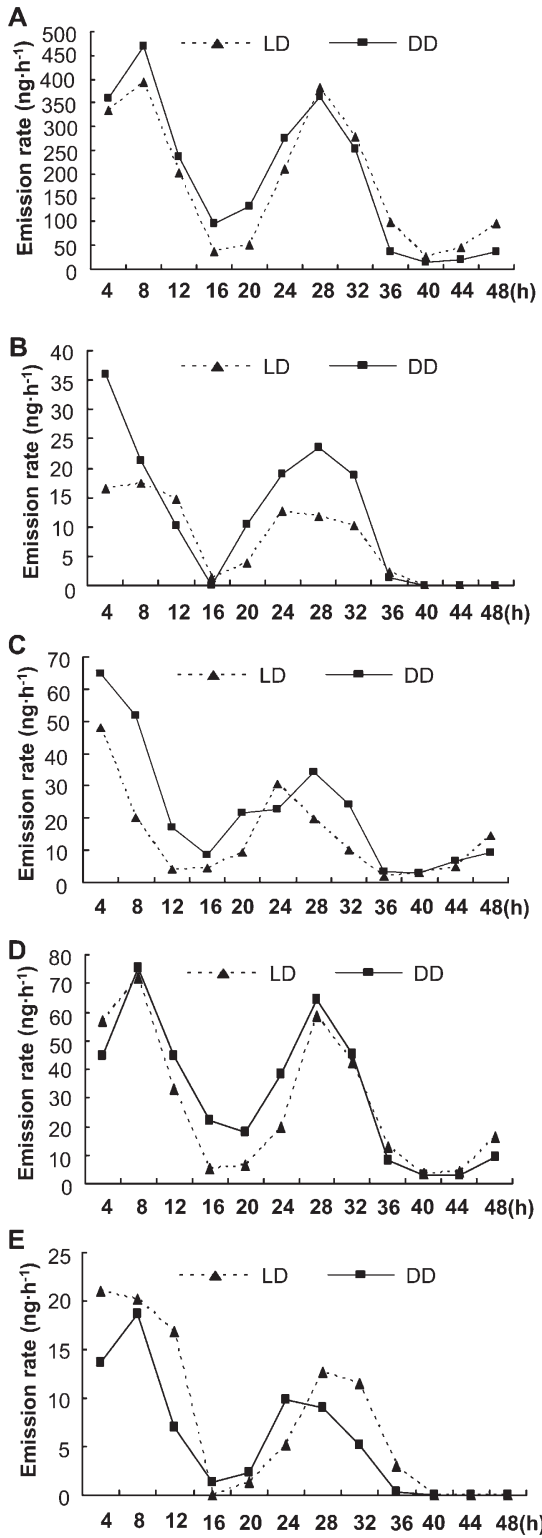

Fig. 2. Emission dynamics of total floral volatiles (A) and individual major volatiles, including (E)- $\beta$-ocimene $(\mathbf{B})$, benzaldehyde $(\mathbf{C})$, geranyl isovalerate (D), and benzyl tiglate (E) from the flowers of the globe amaranth cultivar Fireworks under normal light/dark cycle (LD) and continuous darkness (DD) during a 48-h period. Three replicates yielded similar results. Only emission rates from one representative analysis were presented. The emission rates are presented as nanograms per hour per inflorescence.

isovalerate from the JA-treated flowers and (E)- $\beta$-ocimene, benzyl tiglate, and geranyl isovalerate from the SA-treated flowers were significantly higher than those of control at $4 \mathrm{~h}$ after the treatment (Fig. 3B). In contrast, the emission rates of benzaldehyde, (E)- $\beta$-ocimene, benzyl isovalerate, geranyl propionate, geranyl isovalerate, and benzyl tiglate from both SAand JA-treated flowers were lower than those of control at $24 \mathrm{~h}$ after the treatment (Fig. 3C).

Cluster analysis of floral volatile emission. Multiple plant metabolites as the products of a same enzyme or biochemical pathway often exhibit a similar pattern of accumulation (Yuan et al., 2008). To gain insight into the biosynthesis of floral volatiles of globe amaranth, the emission patterns of six
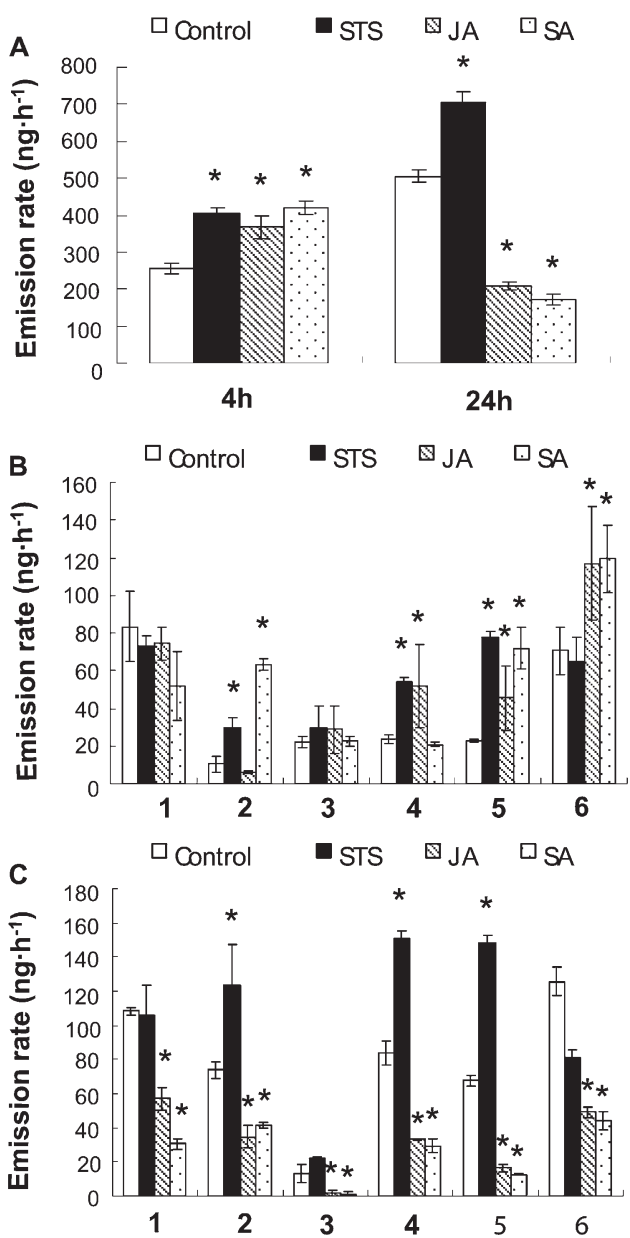

Fig. 3. Emission of total floral volatiles (A) and six major volatile compounds from the control flowers of the globe amaranth cultivar Fireworks and those treated by silver thiosulphate (STS), jasmonic acid (JA), or salicylic acid (SA) at $4 \mathrm{~h} \mathrm{(B)}$ and $24 \mathrm{~h} \mathrm{(C)}$ after the beginning of each treatment. In $\mathbf{B}$ and $\mathbf{C}$, bars 1 through 6 correspond to benzaldehyde, (E)- $\beta$-ocimene, benzyl isovalerate, geranyl propionate, geranyl isovalerate, and benzyl tiglate, respectively. *Statistical difference by Student's $t$ test at $P \leq 0.05$.

major floral volatiles, including benzaldehyde, (E)- $\beta$-ocimene, benzyl isovalerate, geranyl propionate, geranyl isovalerate, and benzyl tiglate, were compared. The emission rates of these six compounds from the flowers of 'Fireworks' under six conditions (control, chemical treatments with STS, SA, and JA, and under light or dark) were subject to cluster analysis. The six compounds were placed into two clades, A and B (Fig. 4). Clade A contains benzaldehyde, benzyl isovalerate and benzyl tiglate, and Clade B contains (E)- $\beta$-ocimene, geranyl propionate, and geranyl isovalerate.

\section{Discussion}

The flowers of 'Las Vegas White', 'Las Vegas Pink', and 'Las Vegas Purple' are scentless, but the flowers of 'Fireworks' have a low level of scent. Our headspace analysis showed that the flowers of all four cultivars of globe amaranth emit volatiles (Fig. 1). The floral volatiles of four cultivars of globe amaranth showed some similarities. Four volatile compounds, including nonanal, decanal, geranyl acetone, and 4,8,12-tetradecatrienal, 5,9,13-trimethyl, were commonly detected (Table 1). Their volatile profiles also showed differences in both the number 


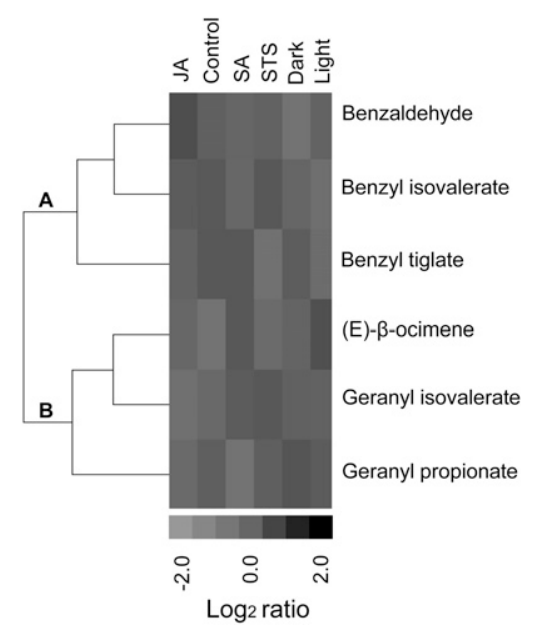

Fig. 4. Cluster analysis of six major floral volatile compounds emitted from the globe amaranth cultivar Fireworks, including benzaldehyde, (E)- $\beta$-ocimene, benzyl isovalerate, geranyl propionate, geranyl isovalerate, and benzyl tiglate. Hierarchical K-means cluster analysis was performed based on emission rate of the chemicals from the flowers of 'Fireworks' under six conditions described in this article that include control, salicylic acid treatment (SA), jasmonic acid treatment (JA), silver thiosulphate treatment (STS), normal light/dark cycle (light), and continuous darkness (dark). The analysis grouped the six compounds into two Clades A and B. of compounds and their quantities. Among 'Las Vegas White', 'Las Vegas Pink', and 'Las Vegas Purple', benzaldehyde is specific to 'Las Vegas Pink'; (E)- $\beta$-ocimene and 3-hexen-1-ol acetate are specific to 'Las Vegas Purple'; and (E)- $\beta$-caryophyllene is specific to 'Las Vegas White'. More prominently, 'Fireworks' flowers emit more volatiles both qualitatively and quantitatively than those of 'Las Vegas White', 'Las Vegas Pink', and 'Las Vegas Purple'. It will be interesting to determine the genetic mechanisms that are responsible for the variations of floral volatiles of four globe amaranth cultivars.

One striking feature of the floral volatile chemistry of 'Fireworks' is the high abundance of esters such as geranyl propionate, geranyl isovalerate, benzyl isovalerate, and benzyl tiglate. These esters can be hypothesized to be formed by the action of acyltransferaseses, especially of those belonging to the acyltransferase (BAHD) family (D'Auria et al., 2007). A number of BAHD acyltransferases were shown to have a broad spectrum of substrate specificity (D'Auria et al., 2002). Determining how many acyltransferases are involved in the production of volatile esters from globe amaranth flowers will be interesting. Theoretically a single acyltransferase can catalyze the formation of all esters (Fig. 5). It becomes more interesting when this possibility is considered together with the result of cluster analysis of globe amaranth floral volatiles. From the cluster analysis (Fig. 4), we can hypothesize that

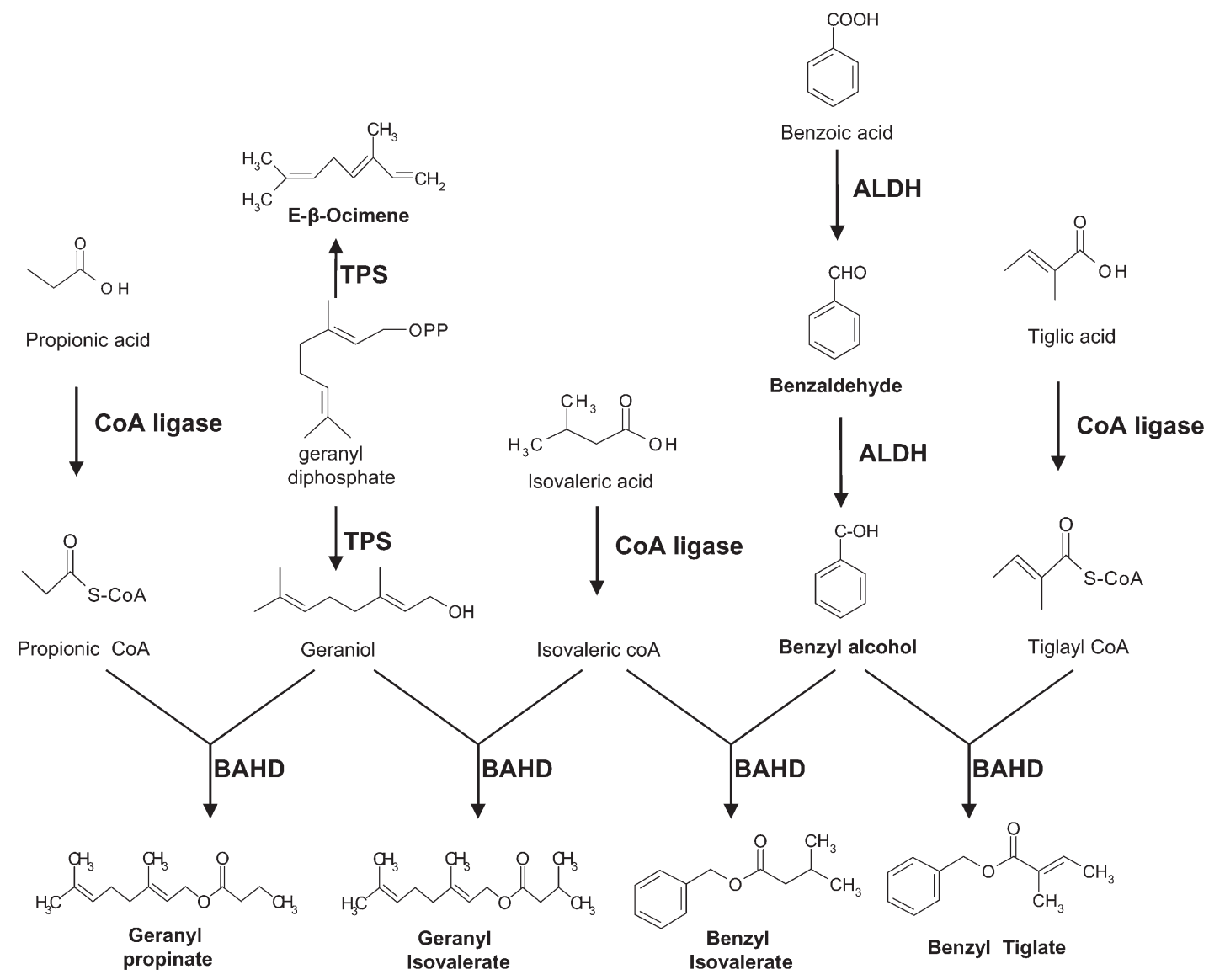

Fig. 5. Putative biochemical pathways leading to the production of six major floral volatiles from the globe amaranth cultivar Fireworks that include benzaldehyde, (E)- $\beta$-ocimene, benzyl isovalerate, geranyl propionate, geranyl isovalerate, and benzyl tiglate. ALDH = aldehyde dehydrogenase; TPS = terpene synthase; BAHD = acyltransferase. 
geranyl propionate and geranyl isovalerate are formed by the action of one acyltransferase and benzaldehyde, benzyl isovalerate, and benzyl tiglate are formed by the action of a separate acyltransferase. Because geranyl propionate and geranyl isovalerate are clustered with (E)- $\beta$-ocimene, an alternative hypothesis is that the formation of these compounds in the two clades is determined by two major biochemical pathways, i.e., the terpenoid pathway for $(\mathrm{E})-\beta$-ocimene, geranyl propionate, and geranyl isovalerate and the benzenoid pathway for benzaldehyde, benzyl isovalerate, and benzyl tiglate. Globe amaranth therefore presents a useful system for studying the biosynthesis and regulation of floral volatiles at the molecular and biochemical levels.

Temporal emission patterns of floral volatiles may be regulated by both internal and external factors. In this study, we found that the emission of floral volatiles from 'Fireworks' exhibited a diurnal pattern (Fig. 2). As reported previously, diurnal emission of floral scent confers an advantage to the host plant by releasing their scent output at maximal levels only when their potential pollinators are active (Galen, 1996) to conserve energy (Dudareva and Pichersky, 2000). Further analysis showed that this diurnal emission of globe amaranth floral volatiles is independent of light and regulated by circadian clock (Fig. 2). We also showed in this study that the emission of floral volatiles can be regulated by phytohormone and defense signaling molecules. STS, an ethylene inhibitor, enhanced the emission of the volatiles derived from the terpenoid pathway such as $(\mathrm{E})-\beta$-ocimene, geranyl propionate, and geranyl isovalerate. Such regulation appears to be pathwayspecific, because the emission of volatiles derived from the benzenoid pathway was not significantly affected by the STS treatment (Fig. 3). JA and SA, two defense signaling molecules, showed temporal effects on the emission of floral volatiles. At $4 \mathrm{~h}$ after the treatment, both chemicals enhanced the emission of volatiles. At $24 \mathrm{~h}$ after the treatment, both chemicals repressed the emission of volatiles (Fig. 3). Although the mechanisms of such regulation is not yet clear, we speculate that exogenous application of JA and SA to the flowers for a short period of time may enhance the release of floral volatiles that can increase the efficacy of pollinator attraction. For the relative longer period of time after the treatment, the emission of volatiles is reduced so the plants may use the energy for defense.

The results presented in this study may lead to several new research directions. One such direction is to study the biological/ecological significance of floral volatiles of globe amaranth. Although aesthetically scentless to humans, the floral volatiles of globe amaranth may play an important role for the reproductive success of this outcrossing species. It was observed that the flowers of the four tested cultivars of globe amaranth were visited by butterflies, bees, and other unidentified insects (Y. Jiang and F. Chen, unpublished results). It will be interesting to determine whether these floral volatiles are involved in attracting pollinators and/or deterring unwanted herbivores. Second, it will be interesting to elucidate the genetic basis that determines floral volatile chemistry of different cultivars of globe amaranth. With the advent of new technology in molecular biology and genomics such as next-generation sequencing, it will be relatively easy to isolate and characterize responsible enzymes and genes (Chen et al., 2009b; Shrivastava et al., 2010). Third, this study has important implications for breeding new cultivars of globe amaranth. Chemically charac- terizing the floral volatiles and identifying and quantifying the critical compounds of the cultivar Fireworks may lay an important foundation for creating new cultivars with distinctive fragrances through plant breeding. Such fragrant cultivars can benefit both the bedding plant and dry flower industry of globe amaranth.

\section{Literature Cited}

Chen, F., H. Al-Ahmad, B. Joyce, N. Zhao, T.G. Kollner, J. Degenhardt, and C.N. Stewart. 2009a. Within-plant distribution and emission of sesquiterpenes from Copaifera officinalis. Plant Physiol. Biochem. 47:1017-1023.

Chen, F., C.J. Liu, T.J. Tschaplinski, and N. Zhao. 2009b. Genomics of secondary metabolism in Populus: Interactions with biotic and abiotic environments. Crit. Rev. Plant Sci. 28:375-392.

Chen, F., J.C. D'Auria, D. Tholl, J.R. Ross, J. Gershenzon, J.P. Noel, and E. Pichersky. 2003. An Arabidopsis thaliana gene for methylsalicylate biosynthesis, identified by a biochemical genomics approach, has a role in defense. Plant J. 36:577-588.

Cocozza Talia, M.A. 1993. Gomphrena globosa and Cirsium japonicum as cut flowers. Colture Protette 22:105-106.

D'Auria, J.C., F. Chen, and E. Pichersky. 2002. Characterization of an acyltransferase capable of synthesizing benzylbenzoate and other volatile esters in flowers and damaged leaves of Clarkia breweri. Plant Physiol. 130:466-476.

D'Auria, J.C., E. Pichersky, A. Schaub, A. Hansel, and J. Gershenzon. 2007. Characterization of a BAHD acyltransferase responsible for producing the green leaf volatile (Z)-3-hexen-1-yl acetate in Arabidopsis thaliana. Plant J. 49:194-207.

Dudareva, N. and E. Pichersky. 2000. Biochemical and molecular genetic aspects of floral scents. Plant Physiol. 122:627-633.

Effmert, U., J. Grosse, U.S.R. Rose, F. Ehrig, R. Kagi, and B. Piechulla. 2005. Volatile composition, emission pattern, and localization of floral scent emission in Mirabilis jalapa (Nyctaginaceae). Amer. J. Bot. 92:2-12.

Euler, M. and I.T. Baldwin. 1996. The chemistry of defense and apparency in the corollas of Nicotiana attenuate. Oecologia 107: $102-112$

Galen, C. 1996. Rates of floral evolution: Adaptation to bumblebee pollination in an alpine wildflower, Polemonium viscosum. Evolution 50:120-125.

Howe, G.A. 2004. Jasmonates as signals in the wound response. J. Plant Growth Regul. 23:223-237.

Kolosova, N., N. Gorenstein, C.M. Kish, and N. Dudareva. 2001. Regulation of circadian methyl benzoate emission in diurnally and nocturnally emitting plants. Plant Cell 13:2333-2347.

Lourdusamy, D.K., E. Vadivel, and R.S.A. Manavalan. 2002. Studies on dyeing of globe amaranth (Gomphrena globosa) for dry flower decoration. South Indian Hortic. 50:645-648.

Matile, P. and R. Altenburger. 1988. Rhythms of fragrance emission in flowers. Planta 174:242-247.

Niu, Y.F., C.M. Han, Y. Shao, and Y.D. Tao. 2009. Comparative study on eight trace elements in twelve flower medicines. Spectroscopy Spectral Anal. 29:1997-2000.

Raguso, R.A. 2004. Why are some floral nectars scented? Ecology 85:1486-1494.

Sexton, R., A.E. Porter, S. Littlejohns, and S.C. Thain. 1995. Effects of diazocyclopentadiene (DACP) and silver thiosulfate (STS) on ethylene regulated abscission of sweet-pea flowers (Lathyrus-odoratus L.). Ann. Bot. (Lond.) 75:337-342.

Shrivastava, G., M. Rogers, A. Wszelaki, D.R. Panthee, and F. Chen. 2010. Plant volatiles-based insect pest management in organic farming. Crit. Rev. Plant Sci. 29:123-133.

Shulaev, V., P. Silverman, and I. Raskin. 1997. Airborne signalling by methyl salicylate in plant pathogen resistance. Nature 385:718721. 
Underwood, B.A., D.M. Tieman, K. Shibuya, R.J. Dexter, H.M. Loucas, A.J. Simkin, C.A. Sims, E.A. Schmelz, H.J. Klee, and D.G. Clark. 2005. Ethylene-regulated floral volatile synthesis in petunia corollas. Plant Physiol. 138:255-266.

Yang, Y., J.S. Yuan, J. Ross, J.P. Noel, E. Pichersky, and F. Chen. 2006. An Arabidopsis thaliana methyltransferase capable of methylating farnesoic acid. Arch. Biochem. Biophys. 448:123132.

Yuan, J.S., T.G. Köllner, G. Wiggins, J. Grant, J. Degenhardt, and F. Chen. 2008. Molecular and genomic basis of volatile-mediated indirect defense against insects in rice. Plant J. 55:491-503.
Zhao, N., J. Guan, J.L. Ferrer, N. Engle, M. Chern, P. Ronald, T.J. Tschaplinski, and F. Chen. 2010. Biosynthesis and emission of insect-induced methyl salicylate and methyl benzoate from rice. Plant Physiol. Biochem. 48:279-287.

Zhao, N., J. Guan, F. Forouhar, T.J. Tschaplinski, Z.M. Cheng, L. Tong, and F. Chen. 2009. Two poplar methyl salicylate esterases display comparable biochemical properties but divergent expression patterns. Phytochemistry 70:32-39.

Zhuang, X.F., W.E. Klingeman, J. Hu, and F. Chen. 2008. Emission of volatile chemicals from flowering dogwood (Cornus florida L.) flowers. J. Agr. Food Chem. 56:9570-9574. 